\title{
Transport and Entrapment of Particles in Steel Continuous Casting
}

\author{
Brian G. Thomas, Quan Yuan, Rui Liu, Sana Mahmood, and Rajneesh Chaudhary \\ University of Illinois at Urbana-Champaign \\ Department of Mechanical Science and Engineering; \\ 1206 W. Green St., Urbana, IL, 61801, U.S.A. \\ Tel: 217-333-6919, Fax: 217-244-6534, Email: bgthomas@illinois.edu
}

Keywords: Continuous Casting, Large Eddy Simulation, particle transport, particle entrapment

\begin{abstract}
A particle-entrapment model based on local force balances has been developed, implemented into computational models of turbulent fluid flow, and applied to simulate the entrapment of slag inclusions and bubbles during the continuous casting of steel slabs. Turbulent flow of molten steel is computed in the nozzle and mold using transient CFD models. Next, the transport and capture of over 30,000 particles are simulated using a Lagrangian approach. Particles touching the dendritic interface may be pushed away, dragged away by the transverse flow, or captured into the solidifying shell according to the results of a local balance of ten different forces. This criterion was validated by reproducing experimental results in two different systems. Finally, the model is applied to predict the entrapment distributions of different sized particles in a typical slab caster. Although more large particles are safely removed than small ones, the capture rate as defects is still high.
\end{abstract}

\section{Introduction}

Surface defects such as slivers and blisters are often caused by captured inclusion clusters, slag, bubbles, and other particles. During continuous casting, jets of molten steel from the submerged entry nozzle (SEN) ports carry bubbles and inclusion particles into the mold cavity from upstream processing, as shown in Figure 1 [1]. In addition, droplets of liquid mold slag may become entrained into the flowing steel due to fluid flow problems, via several different mechanisms [2]. Figure 2 shows some typical inclusion particles [3] that can be entrapped from the flowing liquid into the solidifying steel shell to form defects in continuous-cast product.

Liquid steel flow in the continuous casting mold has been modeled extensively. Reynolds Average Navier-Stokes (RANS and URANS) approaches are based on accurate computation of the ensemble-averaged velocity field. Particle transport can be tracked using a Lagrangian approach, introducing an extra model, such as "random walk" to generate realistic motion of every particle. Large Eddy Simulation (LES) models can accurately calculate both the transient evolving flow field and Lagrangian particle motion $[4,5]$ of this turbulent process.

Having computed the particle paths, a criterion is needed to determine the fate of each particle. Particles reaching the solidification front may either become entrapped into the solidifying shell, or drift away with the liquid steel. Many previous works adopted the simple criterion that each particle is entrapped into the shell if it touches the solidification front [6]. However, this oversimplified criterion over predicts the entrapment of large particles. Yuan and Thomas [7] developed a criterion for particle entrapment into the solidification front, accounting for the effects of particle properties, Primary Dendrite Arm Spacing (PDAS), local flow field, local concentration gradients, surface tension effects, and other forces. This method is summarized here, validated, and applied to predict particle entrapment in realistic continuous-casting flow. 


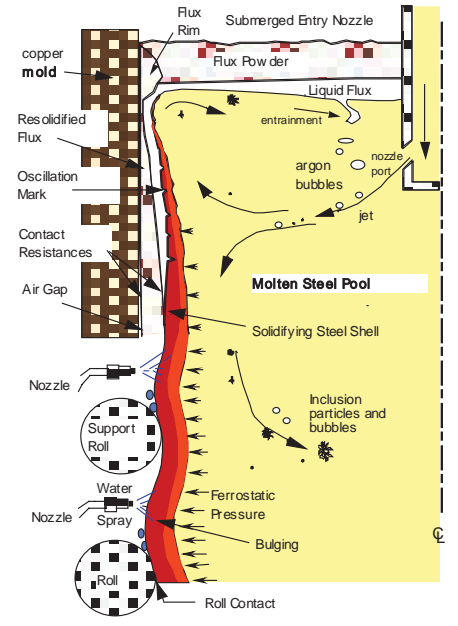

Figure 1. Complex Transport Phenomena in Continuous Casting Mold [1]

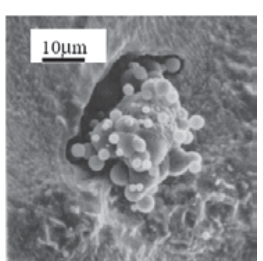

(a) Alumina cluster

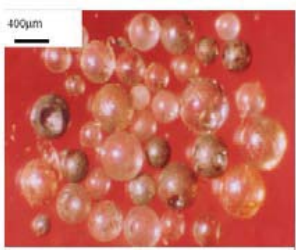

(c) Slag inclusions

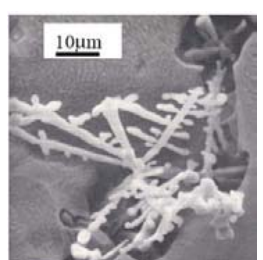

(b) Alumina dendrite

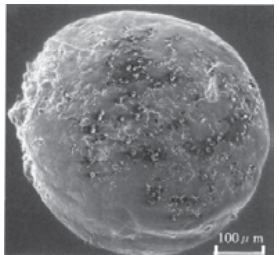

(d) Bubble with inclusions

Figure 2. Different Types of Particles in Continuous Casting Process [3]

\section{Model Description}

Fluid Flow Model

Models are needed for the liquid steel flow field, particle transport and particle entrapment at the solidification front. Modeling the fluid flow requires solving the mass balance (continuity) and momentum equations, and is presented in detail elsewhere [4].

$$
\nabla \cdot(\rho \mathbf{u})=0 \text { and } \frac{\partial(\rho \mathbf{u})}{\partial t}+\nabla \cdot(\rho \mathbf{u u})=-\nabla p+\nabla \cdot(\mu \nabla \mathbf{u})+\mathbf{f}
$$

The flow domains modeled in this work include only the liquid pool, as the walls represent the solidification front. This requires finding the shape of the solid shell using separate models of solidification and stress, which include the interfacial gap and mold [8]. Wall law boundary conditions enforce downward movement at the casting speed, and include mass and momentum sinks $[4,9]$ to account for mass flow across the interface in both the in-house LES code, CUFLOW [4] and in FLUENT [10].

\section{Particle Transport Model}

The motion of inclusion particles can be simulated by integrating the following transport equation for each particle, which considers contributions from seven different forces:

$$
\mathbf{v}=\frac{d \mathbf{x}_{p}}{d t} \text { where } m_{p} \frac{d \mathbf{v}}{d t}=\mathbf{F}_{D}+\mathbf{F}_{L}+\mathbf{F}_{\text {added-mass }}+\mathbf{F}_{G}+\mathbf{F}_{\text {press }}+\mathbf{F}_{\text {stress }}
$$

The terms on the right hand side (RHS) of Eq. (2) are drag force, lift force, added mass force, pressure gradient force, stress gradient force, and gravitational force. A seventh force, Basset history force, was found to be negligible, owing to the small particles $(\leq \sim 100 \mu \mathrm{m})$ of interest in this work [7]. Each of the six hydrodynamic forces in Eq. (2) is discussed next, with further details presented elsewhere [7,9].

The drag force, $\mathbf{F}_{D}$, is calculated in Eq. (3), which also defines the particle Reynolds number 


$$
\mathbf{F}_{D}=\frac{1}{8} \pi d_{p}^{2} \rho_{f} C_{D}|\mathbf{v}-\mathbf{u}|(\mathbf{v}-\mathbf{u}), \text { where } C_{D}=\frac{24}{\operatorname{Re}_{p}}\left(1+0.15 \operatorname{Re}_{p}^{0.687}\right), \operatorname{Re}_{p}=\frac{|\mathbf{v}-\mathbf{u}| d_{p}}{v}
$$

The lift force, $\mathbf{F}_{L}$, is calculated via Eqs. (4) and (5), with $u_{1}$ and $v_{1}$ defined as instantaneous streamwise velocities for liquid and particle, and $\mathrm{G}$ is the wall normal gradient of $u_{l}$ [9].

$$
\begin{gathered}
\mathbf{F}_{L}=-\frac{9}{4 \pi} \mu d_{p}^{2} U_{s} \operatorname{sgn}(G)\left[\frac{|G|}{v}\right]^{\frac{1}{2}} J, \text { where } G=\frac{d u_{1}}{d y}, \varepsilon=\operatorname{sgn}(G) \frac{\sqrt{|G| v}}{U_{s}}, U_{s}=u_{1}-v_{1} \\
\quad \text { and } J(\varepsilon)=0.6765 *\left(1+\tanh \left[2.5 \log _{10} \varepsilon+0.191\right]\right)(0.667+\tanh [6(\varepsilon-0.32)])
\end{gathered}
$$

The added mass force is calculated in Eqs. (6) below:

$$
\mathbf{F}_{\text {added-mass }}=C_{A} \frac{\rho_{f} \pi d_{p}^{3}}{12}\left(\frac{D \mathbf{u}}{D t}-\frac{d \mathbf{v}}{d t}\right), \text { where } C_{A}=2.1-\frac{0.132}{0.12+\mathrm{Ac}^{2}}, \mathrm{Ac}=\frac{|\mathbf{v}-\mathbf{u}|^{2}}{d_{p} \frac{d|\mathbf{v}-\mathbf{u}|}{d t}}
$$

The pressure and stress gradient forces, as well as the gravitational force, are calculated via:

$$
\mathbf{F}_{\text {press }}+\mathbf{F}_{\text {stress }}=-\frac{\pi d_{p}^{3}}{6} \frac{D \mathbf{u}}{D t} \text { and } \mathbf{F}_{G}=\frac{\rho_{p} \pi d_{p}^{3}}{6} \mathbf{g}
$$

Particle Entrapment Model

Extra forces are exerted on particles which get close to the solidification front, including the lubrication force, Van der Waals force, and the surface tension gradient force.

The lubrication force is calculated in Eq. 8,

$$
F_{\text {lub }, n}=6 \pi \mu V_{\text {sol }} \frac{R_{p}^{2}}{h_{0}}\left(\frac{r_{\text {tip }}}{r_{\text {tip }}+R_{p}}\right)^{2}
$$

where $\mu$ is fluid viscosity, $V_{\text {sol }}$ is the solidification front moving velocity, $h_{0}$ is the distance between the dendrite tip and particle, $R_{p}$ is the particle radius, and $r_{t i p}$ is the dendrite tip radius. The Van der Waals force as given as follows:

$$
F_{I}=2 \pi \Delta \sigma_{0} \frac{r_{t i p} R_{p}}{r_{t i p}+R_{p}} \frac{a_{0}^{2}}{h_{0}^{2}}, \text { where } \Delta \sigma_{0}=\sigma_{s p}-\sigma_{s l}-\sigma_{p l}
$$

where $\sigma_{s p}, \sigma_{s l}$, and $\sigma_{p l}$ represent the surface tensions for shell-particle, shell-liquid and particleliquid respectively, and $a_{0}$ is the diameter of a liquid atom.

The surface tension gradient force is calculated with Eqs. (10), (11) and (12), and details of $C^{*}$, $C_{0}, n$, and $V_{\text {sol }}$ can be found in previous work [7].

$$
F_{\text {Grad }}=-\frac{m \beta \pi R_{p}}{\xi^{2}}\left\{\frac{\left(\xi^{2}-R_{p}^{2}\right)}{\beta} \ln \left[\frac{\left(\xi+R_{p}\right)\left[\alpha\left(\xi-R_{p}\right)+\beta\right]}{\left(\xi-R_{p}\right)\left[\alpha\left(\xi+R_{p}\right)+\beta\right]}\right]+\frac{2 R_{p}}{\alpha}-\frac{\beta}{\alpha^{2}} \ln \left[\frac{\alpha\left(\xi+R_{p}\right)+\beta}{\alpha\left(\xi-R_{p}\right)+\beta}\right]\right\}
$$




$$
\begin{gathered}
\text { where } \alpha=1+n C_{0}, \beta=n r_{\text {tip }}\left(C^{*}-C_{0}\right) \text {, and } \xi=R_{p}+r_{\text {tip }}+h_{0} \\
\text { and } C^{*} \text { is calculated from } \quad \frac{V_{\text {sol }} r_{t i p}}{2 D_{s}}=\frac{C^{*}-C_{0}}{C^{*}(1-k)}
\end{gathered}
$$

The directions of these extra forces are shown in Figure 3 (a), which shows a typical particle contacting a typical dendritic front shape [11]. A closeup of the thin liquid film between the dendrite tip and a typical particle of alumina or slag is shown in Figure 3(b). For the particle to remain stationary in this moving reference frame requires fluid to continuously flow through this small gap. If the gap becomes smaller than the critical distance, $\mathrm{h}_{0}$, the dendrite tip can grow around the particle to entrap it.

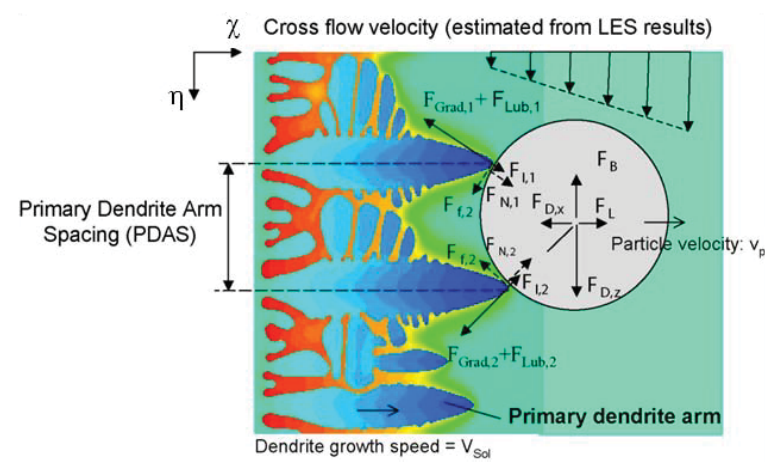

(a) Forces for Particles at Solidification Front

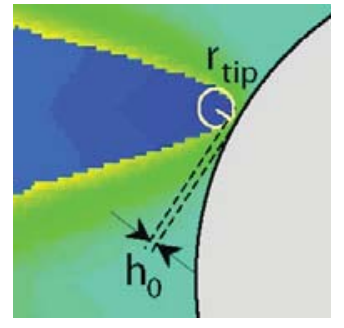

(b) Particle-Dendrite Gap

Figure 3. Force Balance for Particles near Solidification Front [11]

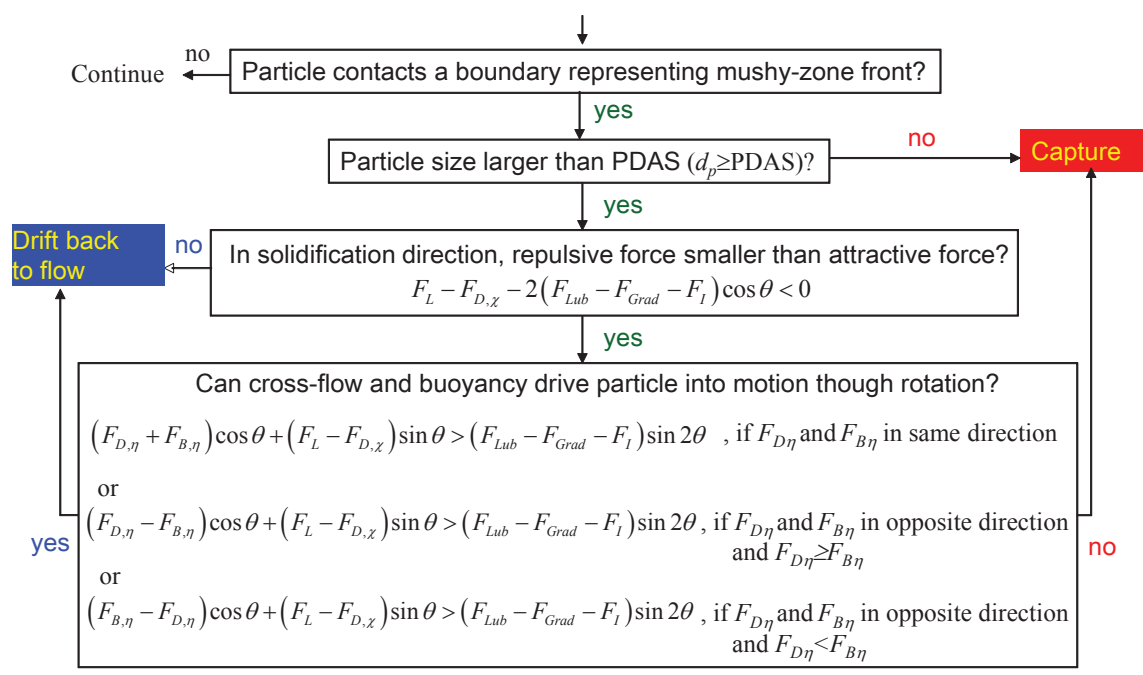

Figure 4. Flow Chart for the Shell Entrapment Criterion [7]

The flow chart used to model particle entrapment is shown in Figure 4. Particles smaller than the local Primary Dendrite Arm Spacing (PDAS), (i.e. $2 \mathrm{R}_{\mathrm{p}}<$ PDAS), can flow between the dendrite arms to become entrapped, whenever they touch the solidification-front boundary in the 
calculation. Particles larger than the local PDAS cannot fit between the dendrite arms. If all of the forces acting on a large particle are in equilibrium, then it will avoid capture by moving with the solidification front that pushes it along in the direction of solidification. This condition is checked by balancing normal force components acting on the particle in the boundary layer region, which include drag force, $F_{D, \chi}$, lift force, and the net effect of lubrication force, Van der Waals force and surface tension gradient force in the normal direction. Usually, however, these repulsive forces in the direction of solidification are not sufficient to push the particle, so the dendrites will grow to surround and capture it, unless the net tangential force acting across the dendrite front causes it to rotate away. This condition is checked by considering a moment balance at dendrite tip, which is dominated by the tangential drag force, $F_{D, \eta}$, and tangential buoyancy force, $F_{B, \eta}$, but also includes lift force, normal drag force, $F_{D, \chi}$, and the net effect of lubrication force, Van der Waals force and surface tension gradient force.

\section{Results and Discussion}

The model described above for particle entrapment at the solidification front was applied to study the inclusion removal rate at top surface based on LES flow simulations, and was validated with plant measurements [7]. Then, particle tracking using the flow field results from the RANS model was performed, for both single-phase and argon-steel two-phase flows. The effects of different particle features and casting conditions on critical downward cross flow velocities and particle removal rate by the top surface were also studied.

Particle Entrapment Model Validation

LES simulation was performed to study the transient flow pattern, particle transport, particle entrapment, and removal rate by the top surface during the continuous casting of a thin slab of 434 stainless steel at $\sim 1.5 \mathrm{~m} / \mathrm{min}$, where extensive water model and plant measurements were available [12]. Sample sections illustrating the predicted flow pattern are given in Figure 5, and shows a classic double-roll recirculating flow pattern. The instantaneous (left) and time-averaged flow pattern (right) are compared, based on $\sim 70$ s of simulation after the flow reached "pseudo"steady state. The observed velocity fluctuations are important to particle dispersal and capture.

The particle transport simulation computes particles touching the top surface slag layer to be removed. Some particles (7\%) touch the nozzle wall. The weighted average of the final removal fractions, given in Table I, is consistent with the results of extensive plant measurements [12], based on slime extraction of many large (500g) samples for typical conditions.

The results shown here suggest that a significant fraction of very large particles can be removed from the mold region. This is known to be the case for straight-walled casters, and is the reason that many companies have invested in changing their top sections from curved to vertical. Alternatively, the less-buoyant, easily-trapped smaller particles always experience small removal fractions. These cause less quality problems owing to their smaller size. Intermediate-sized particles of $100 \mu \mathrm{m}-250 \mu \mathrm{m}$ are large enough to cause severe quality problems, yet are predicted to have high entrapment fractions, even in a vertical caster. Thus, it is important to remove them from the steel during refining stages prior to entering the mold.

\section{Predicted Critical Cross-Flow Velocities in Continuous Steel Caster}

To illustrate the behavior of the validated particle-capture criterion, critical velocities of the flow relative to the downward moving shell for the capture of different particles were computed for different conditions in a steel caster. The flow was assumed to be vertical (upwards or downwards) across a horizontally-growing solidification front, such as encountered near the narrow face in the continuous casting mold region. 


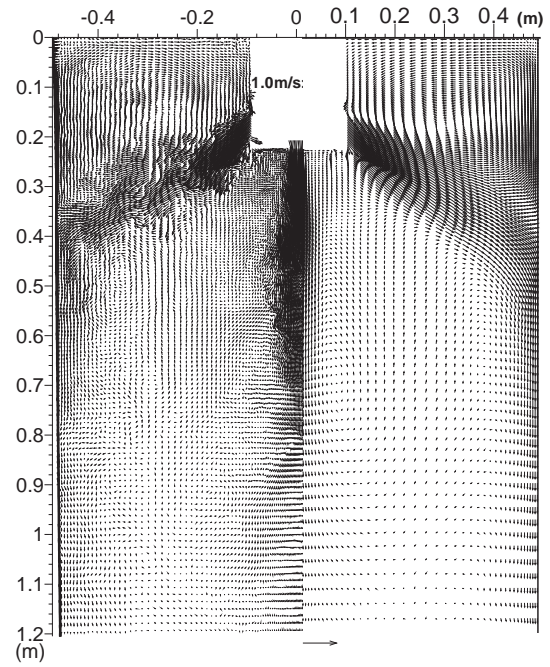

Table I. Particle Removal/Entrapment Rate Calculation vs. Plant Measurement

\begin{tabular}{|c|c|c|}
\hline $\begin{array}{c}\text { Inclusion size } \\
(\boldsymbol{\mu m})\end{array}$ & $\begin{array}{c}\text { Mold slag } \\
\text { (top } \\
\text { surface) }\end{array}$ & $\begin{array}{c}\text { Trapped } \\
\text { in Shell } \\
\text { (product) }\end{array}$ \\
\hline 40 & $7 \%$ & $86 \%$ \\
100 & $12 \%$ & $81 \%$ \\
250 & $42 \%$ & $51 \%$ \\
400 & $70 \%$ & $23 \%$ \\
\hline $\begin{array}{c}\text { Total measured } \\
\text { (tundish to slab) }\end{array}$ & $\mathbf{2 2 \%}$ & $\mathbf{7 8 \%}$ \\
{$[\mathbf{1 2}]$} & & \\
\hline
\end{tabular}

(a) instantaneous flow (b) time average flow

Figure 5. Computed Flow Patterns

The results in Figure 6 show that small particles $(<$ PDAS) are always entrapped, while larger particles are only entrapped within a narrowing window of cross-flow velocity that achieves a force balance. Figure 6 (a) clearly shows that the critical downward cross-flow velocity increases with decreasing particle density. For lighter particles, more downward liquid steel momentum is needed to balance the buoyancy force, in order to keep the particle still at the solidification front and get captured. Figure 6 (b) shows how PDAS affects the critical downward cross flow velocity for capturing argon bubbles. Increasing PDAS traps many more small bubbles, but has less effect on larger bubbles. Bubbles larger than $\sim 600 \mu \mathrm{m}$ are difficult to entrap and are relatively unaffected by PDAS. Figure 6(c) shows little significance of the solidification front moving velocity on critical downward cross flow velocities. Figure 6 (d) shows that sulfur concentration has a more important influence on the critical downward flow velocity. Increasing sulfur especially increases the entrapment of 150-300 $\mu \mathrm{m}$ particles, for the conditions studied.

Effect of multiphase flow on particle entrapment

Liquid steel flow patterns in a typical slab-casting mold for both single-phase and argon-steel two-phase flows are computed using the RANS approach, and 5000 slag droplets are tracked using the Lagrangian approach, with turbulent velocity fluctuations generated with the random walk model. Two different slag droplet diameters, $100 \mu \mathrm{m}$ and $400 \mu \mathrm{m}$, are studied.

Results in Figure 7 (a) and (b) show a dramatic increase in particle removal rate into the top surface as particle size increases. This is because larger particles float faster and are much easier to escape entrapment than smaller particles when they contact the shell. This observation agrees with previous case study with LES. This trend concerning particle size is observed for both single- and two-phase flows. It is also interesting to note more clustering of the entrapment locations of larger particles. This is because only certain regions in the flow field achieve the narrow range of cross-flow velocities at the interface needed to capture larger particles.

Comparing surface removal rates, gas injection increases the top surface removal rate of $100-\mu \mathrm{m}$ diameter particles by a factor of $\sim 5$, relative to single-phase flow. The removal rates of $400-\mu \mathrm{m}$ diameter inclusions stay almost the same. 


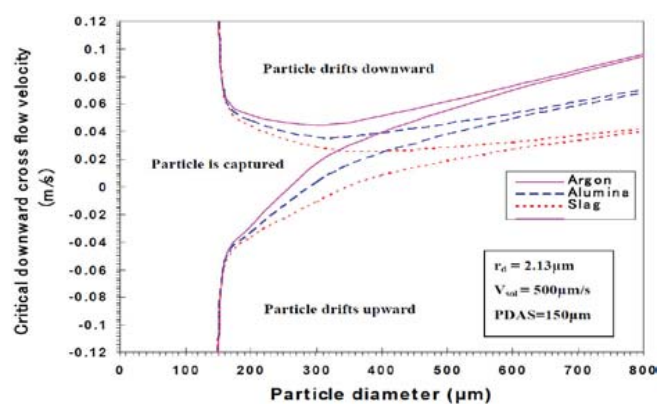

(a) Effect of Particle Types

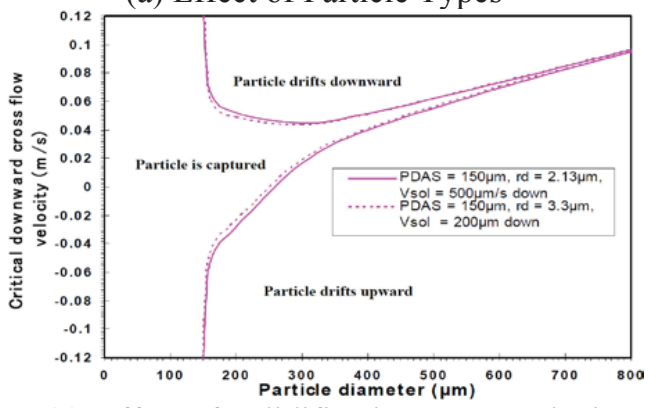

(c) Effect of Solidification Front Velocity, (Argon Bubbles)
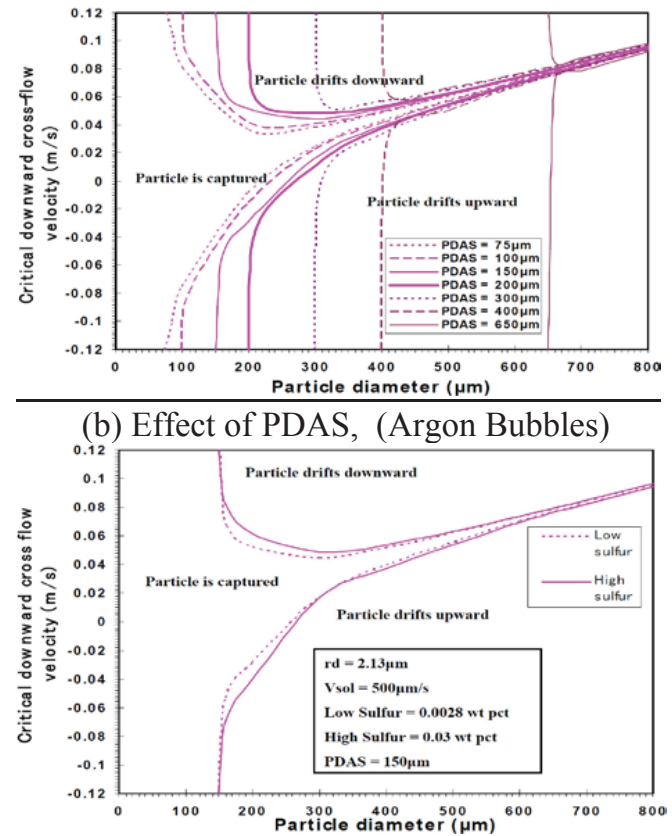

(d) Effect of Sulfur Concentration, (Argon Bubbles)

Figure 6. Effects on Critical Cross-Flow Velocity for Particle Entrapment [9]

\section{Conclusions}

A criterion for particle entrapment in casting processes is presented, which considers normal and tangential force balances involving ten different forces acting on a particle in the boundary layer region near the solidification front. These forces include: transverse drag, (caused by fluid flow across the dendrite interface), gravity (buoyancy), the minor bulk hydrodynamic forces (lift, pressure gradient, stress gradient, Basset, and added mass forces), and other forces acting at the interface (lubrication drag, Van der Waals, and surface tension gradient forces). This capture criterion has been validated with experimental measurements.

Lagrangian computations of particle transport during continuous casting of steel slabs were performed, based on fluid velocity fields obtained from both LES and RANS simulations. Using the particle entrapment model, removal rates to the slag layer were computed. The results reveal:

1. Particle entrapment depends mainly on the particle size and density, transverse fluid velocity, sulfur concentration gradient, solidification front velocity, and PDAS.

2. Increasing the number of particles improves the accuracy of removal predictions, especially for later times (e.g. 10-100s). At least 2500 particles are required to obtain accuracy within $\pm 3 \%$.

3. Overall particle removal rates to the top surface are small, $(<20 \%)$, so upstream refining, prevention of reoxidation, and mold flow patterns to avoid slag entrainment are crucial.

4. Particle removal to the top surface decreases greatly $(\sim 50 \%$ to $\sim 10 \%)$ with decreasing particle size, (400 to $100 \mu \mathrm{m})$ using both LES and RANS modeling of different casters.

5. Gas injection helps to increase the particle removal rate at top surface for relatively small particles $(\sim 100 \mu \mathrm{m})$, but provides not much improvement for larger particles $(\sim 400 \mu \mathrm{m})$. 


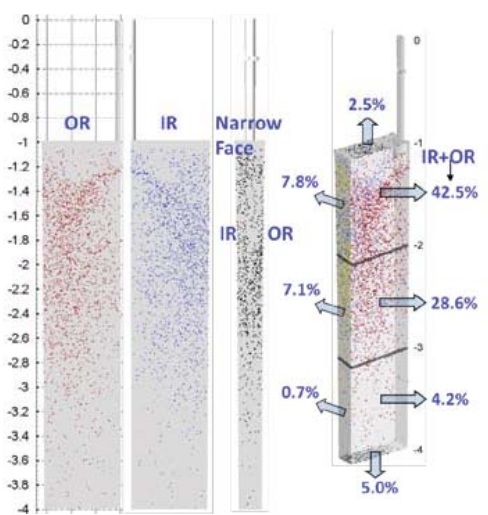

(a) Single phase flow, $100 \mu \mathrm{m}$ slag particle

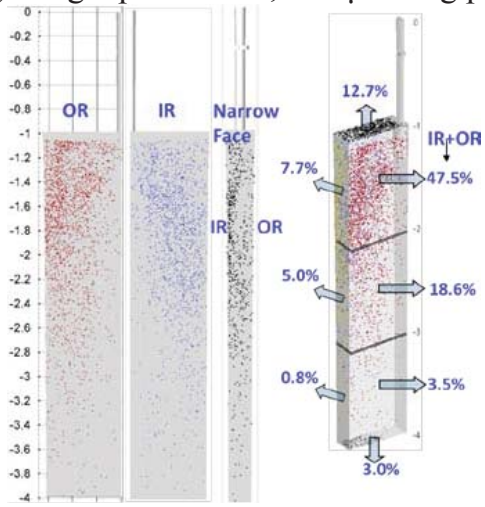

(c) Argon-steel flow, $100 \mu \mathrm{m}$ slag particle

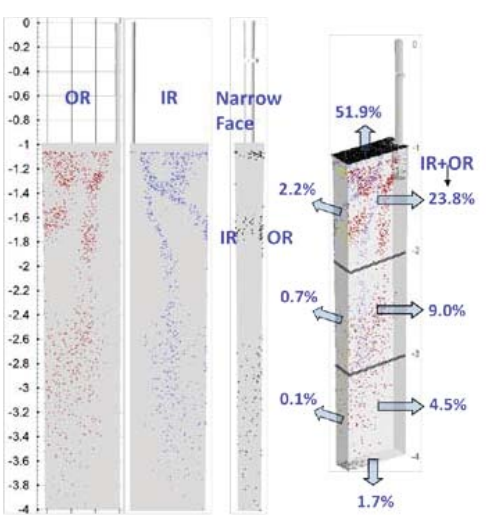

(b) Single phase, $400 \mu \mathrm{m}$ slag particle

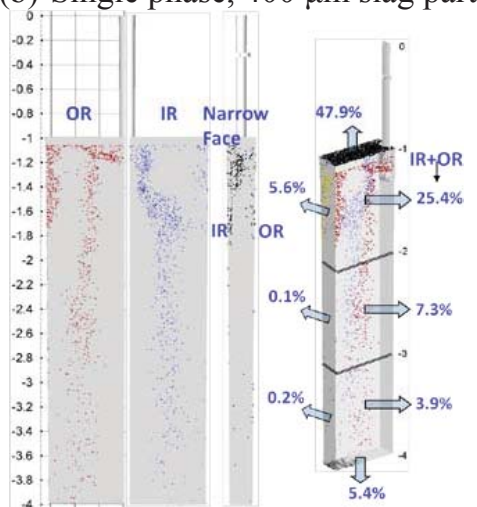

(d) Argon-steel flow, $400 \mu \mathrm{m}$ slag particle

Figure 7. Effects of flow pattern and particle size on particle distribution in the caster

\section{References}

1. Y. Meng and B.G. Thomas, "Heat Transfer and Solidification Model of Continuous Slab Casting: Con1d", Metall. Mater. Trans. B, 34B(5), (2003), 685-705.

2. L. Hibbeler and B.G. Thomas, "Investigation of Mold Flux Entrainment in CC Molds Due to Shear Layer Instability", AISTech 2010 Steelmaking Conference Proc., Pittsburgh, (2010).

3. L. Zhang and B.G. Thomas, "State of the Art in Evaluation and Control of Steel Cleanliness", ISIJ International, 43(3), (2003), 271-291

4. Q. Yuan, B.G. Thomas and S.P. Vanka, "Study of Transient Flow and Particle Transport during Continuous Casting of Steel Slabs, Part 1. Fluid Flow", Metal. Mater. Trans. B., 35B (4), (2004), 685-702.

5. Q. Yuan, B. G. Thomas, and S. P. Vanka, "Study of Transient Flow and Particle Transport during Continuous Casting of Steel Slabs, Part 2. Particle Transport", Metal. Mater. Trans. B., 35B (4), (2004), 703-714

6. Y. Wang and L. Zhang, "Fluid Flow-Related Transport Phenomena in Steel Slab Continuous Casting Strands under Electromagnetic Brake", Metal. Mater. Trans. B., (2011), 1-33.

7. Q. Yuan and B.G. Thomas, "Transport and Entrapment of Particles in Continuous Casting of Steel," 3rd International Congress on Science \& Technology of Steelmaking, Charlotte, NC, May 9-12, (2005), 745-762.

8. S. Koric, L. Hibbeler, R. Liu and B.G. Thomas, "Multiphysics Model of Metal Solidification on the Continuum Level", Numerical Heat Transfer, Part B: Fundamentals, 58(6), (2010), 371-392.

9. S. Mahmood, "Modeling of Flow Asymmetries and Particle Entrapment in Nozzle and Mold during Continuous Casting of Steel Slabs", MS thesis, Mech. Sci. \& Engng, University of Illinois at Urbana-Champaign, (2006).

10.FLUENANSYS Inc., FLUENT6.3-Manual, 2007, ANSYS Inc., Lebanon,NH.

11.J-H.Jeong, N. Goldenfeld and J.A. Dantzig, Physical Review E, (64), (2001), 1-14.

12. L. Zhang, S. Yang, K. Cai, J. Li, X. Wan and B. G. Thomas, "Investigation of Fluid Flow and Steel Cleanliness in the Continuous Casting Strand”, Metal. Mater. Trans B., 38B(1), (2007), 63-83. 